\title{
Effect of a Four-Week Self-Administered Acupressure Intervention on Perceived Stress over the Past Month
}

\author{
Yasuhiro Honda, Akira Tsuda, Satoshi Horiuchi \\ Kurume University, Kurume, Japan \\ Email: ya-honda@dmr.ac.jp
}

Received March 16, 2012; revised April 19, 2012; accepted May 17, 2012

\begin{abstract}
The use of relaxation techniques in daily life is an effective means for the self-management of stress. Acupressure is a traditional technique where pressure is applied to acupuncture points instead of puncturing the skin. Self-administered acupressure is a potential method for dealing with stress. The effect of self-administered acupressure on anxiety has been examined but whether it can reduce perceived stress over longer periods is unknown. This study aimed to examine whether a self-administered, four-week acupressure intervention would reduce perceived stress over the past month. Fifteen male and nine female college students (age, $28.9 \pm 8.51 \mathrm{yr}$ ) majoring in acupuncture and moxibustion medicine were randomly assigned to self-acupressure (AG) and control groups (CG). AG participants were instructed to conduct five sessions of acupressure in the morning, midday, and night. Each session included pressing six acupressure points on the neck (three points on the left and right side each) for five seconds. CG participants were asked to spend their daily life as usual. The outcome was the perceived stress level during the past month, which was assessed using a reliable and valid four-item scale. Perceived stress was measured at baseline, two weeks later, and after intervention. The stress level did not significantly differ between the two groups at baseline. In the AG, the stress level decreased from baseline to two weeks later and remained constant until the end of intervention. The stress level was significantly lower in the AG than in the CG only after intervention. These results provided initial evidence that self-administered acupressure reduces perceived stress over the past month.
\end{abstract}

Keywords: Self-Administered Acupressure; Perceived Stress; Self-Care; Relaxation; Stress Management

\section{Introduction}

Perceived stress has negative effects not only on mental and physical health but also on productivity [1,2]. With a primary prevention focus, it is important to manage stress by oneself [3]. Despite this, at least one in four American adults does not engage in healthy activities to manage stress [4]. This is also true of Korean [5] and Japanese college students [6]. Thus, it is important to explore useful tools for the self-management of perceived stress, which could prevent stress-related health problems.

One approach to the self-management of perceived stress is the use of relaxation techniques in daily life. Major techniques include autogenic training, progressive relaxation, and biofeedback. However, no relaxation technique has been shown to be effective in all individuals. Moreover, it is important to determine whether other relaxation techniques are effective [7]. This evaluation will aid in designing relaxation techniques that suit each individual's specific needs.

A well-known relaxation technique is acupressure. This treatment method is used in Chinese medicine. The meridian theory assumes that energy (Qi) flows through meridians, which are invisible circuitries of channels in the body. The occurrence of Qi imbalance, i.e., if the energy flow is too slow, fast, turbulent, or static, mental and physical health is disturbed. Acupressure involves the application of pressure on the traditional acupuncture points instead of puncturing the skin, and aims to correct such imbalances. Previous studies have suggested that acupressure is an effective method for reducing stress [8]; anxiety [9]; depressive mood [10] as well as improving general health [11].

Acupressure can be self-administered if users are trained to press certain acupuncture points accurately. Self-administered acupressure is cost-free and simple because it only requires the pressing of acupuncture points. Thus, we suggest that self-administered acupressure could be an effective tool for stress self-management.

Despite the potential of this method, very few studies have examined the effects of self-acupressure on perceived stress and anxiety [12]. Among these, Chen and Chen [12] reported that the application of self-acupres- 
sure on the Sanyinjiao point (acupuncture point located above the ankle) did not reduce anxiety when it was measured using a visual analogue scale. They suggested that anxiety may have not been reduced because the anxiety level was already low. Das, Nayak, and Margaret [13] reported that self-administered acupressure on Neiguan point, Yangbai point, and Yintang point was effective in reducing physical stress in high school students. The design was, however, quasi-experimental. In addition, self-administered acupressure has been applied to the management of smoking [14], persistent cancerrelated fatigue [15], pain associated with dysmenorrhea $[12,16]$, relief of dyspnea [17], and pruritus and lichenification [18]. No study has evaluated the effects of selfadministered acupressure on perceived stress for longer periods such as one week or one month in a randomized controlled trial. From a stress management perspective, it is important to manage perceived stress that is relatively stable over time. Demonstrating that self-administered acupressure reduces perceived stress level over longer periods will support the use of self-administered acupressure as a new relaxation technique.

This study evaluated whether a four-week self-administered acupressure intervention would reduce perceived stress. We hypothesized that perceived stress levels would decrease only in the participants who engaged in self-administered acupressure.

\section{Method}

This study was initiated in the middle of January and ended in February 2012. The final examination of the semester was scheduled at the end of February. The consent form and experimental protocol were approved by the Ethical Committee at Kurume University, Fukuoka, Japan. To test the efficacy of self-administered acupressure, we selected college students majoring in acupuncture and moxibustion medicine as the study's participants. The participants were considered suitable for an initial evaluation of the efficacy of self-administered acupressure because of the following reasons. Acupressure requires that the acupuncture points are pressed accurately; theoretically, its efficacy depends on how accurately each participant presses the points. These participants were expected to have greater knowledge of the positions of the acupuncture points compared with students majoring in other subjects. In addition, it was convenient to select these students because the first author of this paper was a member of the teaching staff in a college teaching this subject.

We asked 21 men and 11 women to participate in this study, but six men and two women refused. Finally, 15 Japanese male and nine female college students agreed to participate in this study, and they provided complete data.
The mean age was 28.9 years $(\mathrm{SD}=8.5)$. We confirmed that all participants had no history of current or previous physical or psychiatric illnesses and that they received no medication. Because of the lack of published data, we were unable to determine how representative they were as a sample of Japanese college students majoring in acupuncture and moxibustion medicine.

The outcome measure in this trial was the perceived stress level, which was measured using the Stress subscale of the Japanese language version of the Rhode Island Stress and Coping Inventory (J-RISCI) [19]. The subscale consists of four items and reflects how often a person experiences stress during the previous month. Each respondent rated how often they experienced stress during the past month using a 5-point Likert scale (1 = never and 5 = repeatedly). The score ranged from 4 to 20 . This measure was found to be reliable and valid [19]. Internal consistency reliability was evaluated using Cronbach's alpha coefficient and found to be acceptable ( $\alpha=$ 0.78). The measure was validated for depressive mood, which was measured using the Japanese version of the Center for Epidemiologic Studies for Depression Scale [20]. Perceived stress is associated with a depressive mood. The J-RISCI stress score was significantly and positively associated with the depressive mood score ( $\mathrm{r}=$ $0.61, \mathrm{p}<0.01$ ), and its validity was supported.

The participants were randomly assigned to a selfacupressure group (AG) and a control group (CG). We first selected 12 students and assigned them to AG, while the remaining students were assigned to CG. AG participants were instructed by the first author to complete five self-acupressure sessions during the morning, midday, and night. Each session involved pressing six acupressure points on the neck (three points on the left and right sides each) for five seconds. The order in which the points were pressed depended on the participants. How strongly these points were pressed was not defined objectively. Instead, the participants were asked to press the points with sufficient pressure such that they felt comfortable. The first author provided a weekly message of encouragement to the AG participants during his lectures on the performance of self-administered acupressure. The CG participants were asked to continue with their daily life as usual, with no change in their life style. No incentive was given to the participants. The J-RISCI Stress subscale was tested at baseline, two weeks later, and after intervention.

We selected three acupuncture points (Figure 1), which are known as GB12, SI17, and LI18, according to the World Health Organization Regional Office in the Western Pacific [21]. These points were selected based on several considerations. We aimed to reduce the perceived stress level by improving the overall flow of Qi. The main meridians of the body pass through the neck, 


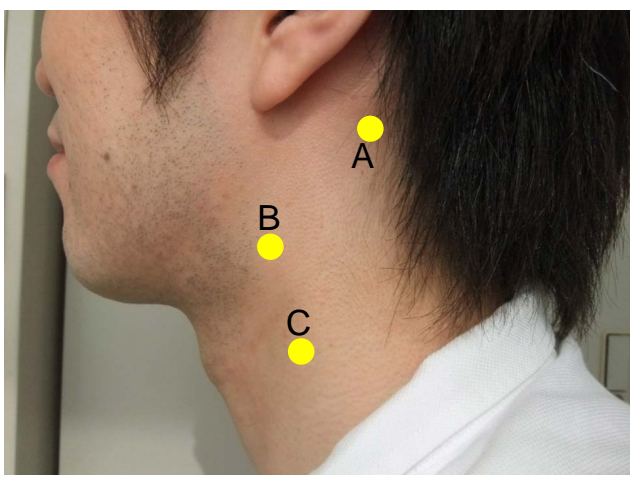

Figure 1. The three acupuncture points on the left side of the neck that each participant was requested to press. Note: $A$, B, and C represent the GB12, SI17, and LI18 points, respectively.

and these three points were located on the main meridians. Thus, it was expected that the application of acupressure on these three points would improve the overall flow of Qi and reduce the perceived stress level.

\section{Results}

Figure 2 shows the stress scores for AG and CG participants at three assessment points (baseline, two weeks later, and after intervention). Analysis of variance with period as a within-subject factor and group as betweensubject factor detected a significant effect of interaction $[\mathrm{F}(2,44)=6.48, \mathrm{p}<0.01]$. There were no significant main effects of period $[\mathrm{F}(1,22)=0.64$, n.s. $]$ and group $[F(2,44)=0.64$, n.s. $]$. Analyses of the interaction revealed significant effects of period in the $\operatorname{AG}[F(2,44)=$ 5.36, $\mathrm{p}<0.01]$, but not in the CG $[\mathrm{F}(2,44)=1.76$, n.s. $]$. These results indicated that the stress level decreased significantly from baseline to two weeks later in the AG, and it remained constant until the end of intervention ( $\mathrm{p}$ $<0.05$ ). There was also a significant effect of group at the end of intervention $[\mathrm{F}(1,22)=4.32, \mathrm{p}<0.05]$, but not before it $[\mathrm{F}(1,22)=0.85$, n.s. $]$ and two weeks later $[\mathrm{F}(1,22)=1.98$, n.s. $]$.

\section{Discussion}

This study evaluated whether a four-week self-administered acupressure course would reduce the perceived stress level. The stress score decreased significantly in the AG; it was significantly lower in the AG than in the CG after intervention.

No significant stress-reducing effect of intervention was evident at the middle of intervention, but a significant effect was observed after the four-week intervention. One possible explanation for the absence of any significant effect at the middle of intervention was that the outcome measure was the perceived level of stress during the previous month, and the effect of intervention was not clearly evident two weeks after intervention. The results demonstrated that the application of self-administered acupressure on the GB12, SI17, and LI18 points could be an effective tool for the self-management of perceived stress. This study provides preliminary evidence that self-administered acupressure can reduce the perceived stress level over the past month.

Our findings provided useful information with regard to stress management for college students by demonstrating that self-administered acupressure provides a new option for relaxation. No single relaxation technique has been demonstrated to be effective in all individuals; therefore, a range of stress management interventions meeting an individual's specific needs should be available. Thus, the provision of a new relaxation technique and demonstration of its efficacy are useful. Self-administered acupressure is a simple and cost-free method for inducing a relaxation response, i.e., reduced sympathetic activity. Chang and Jun [21] reported that college students who received a 20 min acupressure session had a significantly lower blood noradrenaline level, which is an indicator of sympathetic activity, $30 \mathrm{~min}$ after the intervention compared with the controls. In addition, acupressure does not interfere with other stress management techniques such as cognitive reframing. Thus, self-administered acupressure can be a useful relaxation tech-

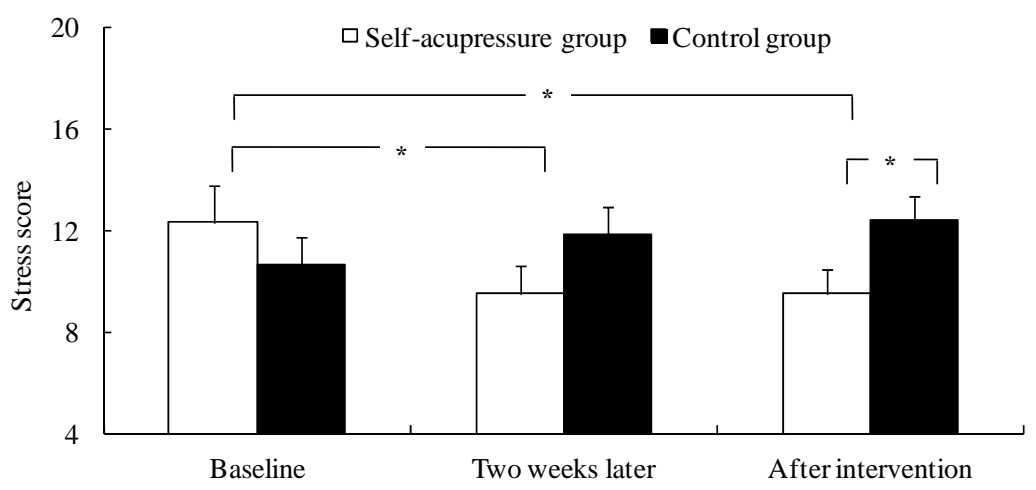

Figure 2. Differences in the perceived stress level scores of the self-acupressure and control groups at baseline, after two weeks, and after intervention. ${ }^{*} \mathbf{p}<0.05$. 
nique and can be incorporated into stress management practices.

This study focused on the perceived stress levels of college students. Stress management is important in this population. Graduating from high school and entering college dramatically affects many aspects of daily life, and most students experience perceived stress [7,22]. Greenberg [22] indicated that stress is perceived as one of the most important health problems experienced by college students, and it is necessary to help them to manage their stress. Thus, a significant decrease in the perceived stress levels of college students is important from a school health perspective.

The main limitation of this study was that the participants were college students who were studying acupuncture medicine. It is necessary to extend our findings to other college students and populations. This extension would be very important because our participants were expected to have more extensive knowledge of the acupuncture points compared with college students majoring in other subjects. Furthermore, it is necessary to replicate our findings in populations other than college students. Another limitation is that the participants may have had some pressure to support the hypothesis because they were also students of one of the authors. Thus, It is necessary to consider such a possibility and make an effort to exclude it.

\section{Acknowledgements}

This work was supported by Grant-in-Aid for Scientific Research (B) (22330196) to AT. The authors would like to thank Enago (www.enago.jp) for the English language review.

\section{REFERENCES}

[1] M. S. Kopp, Á. Skrabski, A. Székely, A. Stauder and R. Williams, "Chronic Stress and Social Changes, Socioeconomic Determination of Chronic Stress," Annals of the New York Academy of Sciences, Vol. 1113, 2007, pp. 325-338. doi:10.1196/annals.1391.006

[2] J. Watts and N. Robertson, "Burnout in University Teaching Staff: A Systematic Literature Review,” Educational Research, Vol. 53, No. 1, 2011, pp. 33-50. doi:10.1080/00131881.2011.552235

[3] K. E. Evers, J. O. Prochaska, J. L. Johnson, L. M. Mauriello, J. A. Padula and J. M. Prochaska, "A Randomized Clinical Trial of a Population- and Transtheoretical Model-Based Stress-Management intervention,” Health Psychology, Vol. 25, No. 4, 2006, pp. 521-529. doi:10.1037/0278-6133.25.4.521

[4] M. L. Robbins, J. L. Fava, G. J. Norman, W. F. Velicer, C. A. Redding and D. A. Levesque, "Stage of Change for Stress Management in Three Samples,” Paper Presented at Nineteenth Annual Scientific Sessions of the Society of
Behavioral Medicine, New Orleans, 1999.

[5] S. Horiuchi, A. Tsuda, E. Kim, K.-S. Hong, Y.-S. Park and U. Kim, "Relationships between Stage of Change for Stress Management Behavior and Perceived Stress and Coping,” Japanese Psychological Research, Vol. 52, No. 4, 2010, pp. 291-297. doi:10.1111/j.1468-5884.2010.00444.x

[6] S. Horiuchi, A. Tsuda, H. Kobayashi and J. M. Prochaska, "The Reliability and Validity of the Japanese Version of Pro-Change's Decisional Balance Measure for Effective Stress Management (PDSM)," Japanese Psychological Research, Vol. 54, No. 2, 2012, pp. 128-136. doi:10.1111/j.1468-5884.2011.00490.x

[7] J. S. Greenberg, "Comprehensive Stress Management," 12th Edition, McGraw-Hill Companies, New York, 2010.

[8] A. Fassoulaki, A. Paraskeva, K. Patris, T. Pourgiezi and G. Kostopanagiotou, "Pressure Applied on the Extra 1 Acupuncture Point Reduces Bispectral Index Values and Stress in Volunteers," Anesthesia \& Analgesia, Vol. 96, No. 3, 2003, pp. 885-890.

[9] A. Kober, et al., "Auricular Acupressure as a Treatment for Anxiety in Prehospital Transport Settings,” Anesthesiology, Vol. 98, No. 6, 2003, pp. 1328-1332. doi:10.1097/00000542-200306000-00005

[10] H. S. Kang, S. R. Sok and J. S. Kang, "Effects of Meridian Acupressure for Stroke Patients in Korea," Journal of Clinical Nursing, Vol. 18, No. 15, 2009, pp. 2145-2152.

[11] F. Kashefi, M. Khajehei, A. R. Ashraf and P. Jafari, “The Efficacy of Acupressure at the Sanyinjiao Point in the Improvement of Women's General Health,” Journal of Alternative and Complementary Medicine, Vol. 17, No. 12, 2011, pp. 1141-1147. doi:10.1089/acm.2010.0589

[12] H. M. Chen and C. H. Chen, "Effects of Acupressure at the Sanyinjiao Point on Primary Dysmenorrhoea," Journal of Advanced Nursing, Vol. 48, No. 4, 2004, pp. 380387. doi:10.1111/j.1365-2648.2004.03236.x

[13] R. Das, B. S. Nayak and B. Margaret, "Acupressure and Physical Stress among High School Students," Holistic Nursing Practice, Vol. 25, No. 2, 2011, pp. 97-104.

[14] L. Leung, T. Neufeld and S. Marin, "Effect of Self-Administered Auricular Acupressure on Smoking Cessation: A Pilot Study," BMC Complementary and Alternative Medicine, Vol. 12, No. 1, 2012, p. 11. doi:10.1186/1472-6882-12-11

[15] S. M. Zick, S. Alrawi, G. Merel, B. Burris, A. Sen, A. Litzinger and R. E. Harris, "Relaxation Acupressure Reduces Persistent Cancer-Related Fatigue,” EvidenceBased Complementary and Alternative Medicine, 2011, Article ID 142913. doi:10.1155/2011/142913

[16] C. L. Wong, K. Y. Lai and H. M. Tse, "Effects of SP6 Acupressure on Pain and Menstrual Distress in Young Women with Dysmenorrheal," Complementary Therapy in Clinical Practice, Vol. 16, No. 2, 2010, pp. 64-69.

[17] S. H. Maa, D. Gauthier and M. Turner, "Acupressure as an Adjunct to a Pulmonary Rehabilitation Program," Journal of Cardiopulmonary Rehabilitation, Vol. 17, No. 4, 1997, pp. 268-276. doi:10.1097/00008483-199707000-00008 
[18] K. C. Lee, et al., "Effectiveness of Acupressure on Pruritus and Lichenification Associated with Atopic Dermatitis: A Pilot Study," Acupuncture Medicine, Vol. 30, No. 1, 2012, pp. 8-11. doi:10.1136/acupmed-2011-010088

[19] S. Horiuchi, A. Tsuda, Y. Tanaka, J. Yajima and S. Tsuda, "Development of the Japanese Version of the Rhode Island Stress and Coping Inventory: Examination of Reliability and Validity," Behavioral Science Research, Vol. 47, No. 1, 2008, pp. 37-46 (in Japanese).

[20] S. Shima, T. Shikano, T. Kitamura and M. Asai, “A New
Self-Report Depression Scale,” Psychiatry, Vol. 27, No. 6, 1985, pp. 717-723 (in Japanese).

[21] S. B. Chang and E. M. Jun, "Effects of SP-6 Acupressure on Dysmenorrhea, Cortisol, Epinephrine, and Norepinephrine in the College Students," Taehan Kanho Hakhoe Chi, Vol. 33, Vol. 7, 2003, pp. 1038-1046 (in Korean).

[22] J. S. Greenberg, "A Study of the Effects of Stress on the Health of College Students: Implications for School Health Education," Health Education Research, Vol. 15, No. 5, 1984, pp. 11-15. 